\title{
Evaluation of the Malaria Surveillance System in Kaduna State, Nigeria 2016
}

\author{
Baffa S. Ibrahim ${ }^{\star 2}$, Aisha A. Abubakar ${ }^{1}$, Ummulkhulthum A. BAJOGA $^{2}$ and \\ Patrick M. Nguku
}

${ }^{1}$ Ahmadu Bello University, Zaria, Zaria, Nigeria; ${ }^{2}$ Nigeria Field Epidemiology and Laboratory Training Program, Abuja, Nigeria

\section{Objective}

To describe the process of operation of the system and assess its key attributes, to determine the effectiveness and efficiency of the surveillance system and make appropriate recommendations to stakeholders for its improvement.

\section{Introduction}

Malaria is a parasitic disease caused by Plasmodium falciparum. About 3.2 billion people worldwide are at risk of malaria. ${ }^{1}$ Children and pregnant women are particularly vulnerable to the disease. SubSaharan Africa carries a high share of the global malaria burden. ${ }^{2}$ Effective malaria surveillance system is essential in the control and elimination of malaria. Worldwide, there were an estimated 198 million cases of malaria in 2013 and 584,000 deaths. ${ }^{1,3,4}$

\section{Methods}

This study was conducted using the "CDC's Updated Guidelines for Evaluating Public Health Surveillance System, 2001". Key stakeholders and Malaria Focal Persons were interviewed. Integrated Disease Surveillance and Response case summary data from January to December 2014 was reviewed. Data analysis was done using Microsoft Excel 2016 and Epi-info 7.

\section{Results}

The system provides information on malaria trends, morbidity and mortality. Case definitions are well understood by participants. All Malaria focal persons (MFPs) were willing to continue using the system. Standardized data collection tools are available in $91 \%$ of Health Facilities (HF). The system was rated flexible by $91 \%$ of MFPs. The system was however not representative because data were essentially from public health facilities only. The system has an average timeliness of $37.7 \%$ and completeness of $59.4 \%$, both parameters were below the State's 80\% target. About 91\% MFPs had refresher training, while 78\% MFPs received supportive supervision. Main challenges identified were lack of commodities in all HFs, and inadequate mobile facilities in $70 \%$ of HFs.

\section{Conclusions}

The Kaduna state Malaria surveillance system is meeting its objectives. However, challenges are observed in its timeliness, representativeness, and data quality. Efforts should be made to integrate tertiary and private health facilities into the system. MFPs need more training on malaria reporting to improve timeliness and data quality. There is the need to improve on the supply of malaria treatment commodities to all health facilities within Kaduna state.
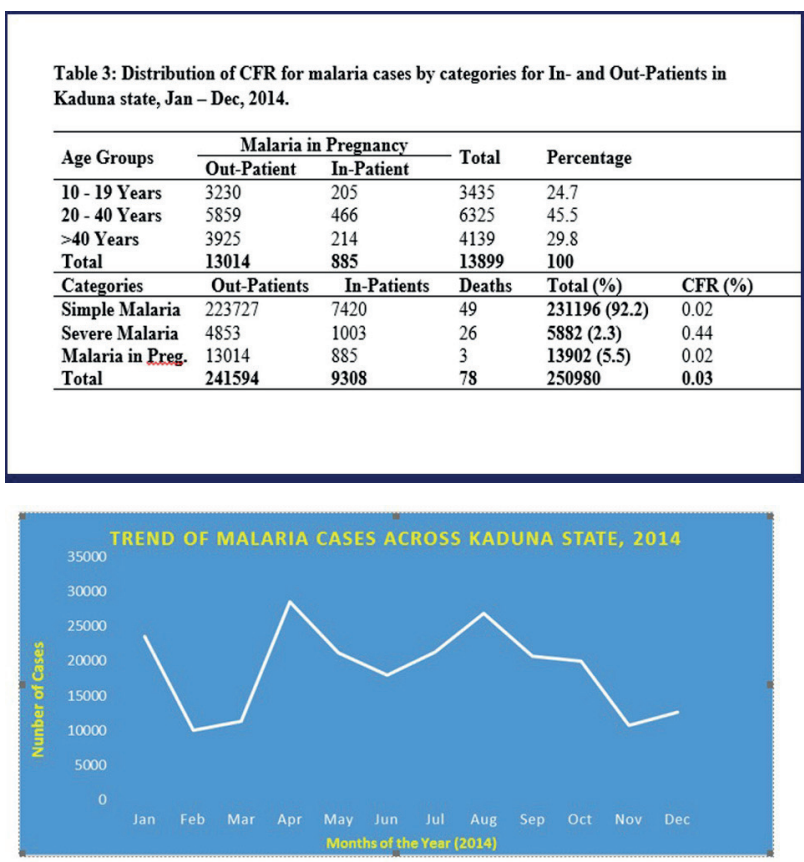

Figure 2: Graph showing the trend of malaria cases across Kaduna state from Jan - Dec, 2014.

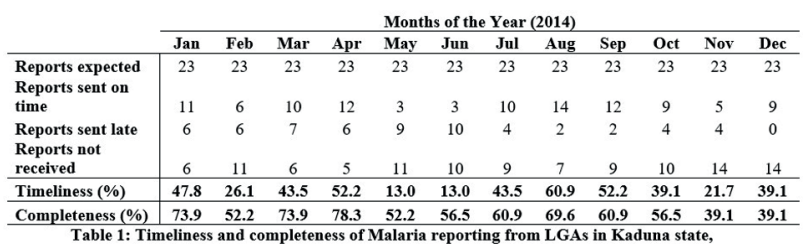

Jan - Dec, 2014

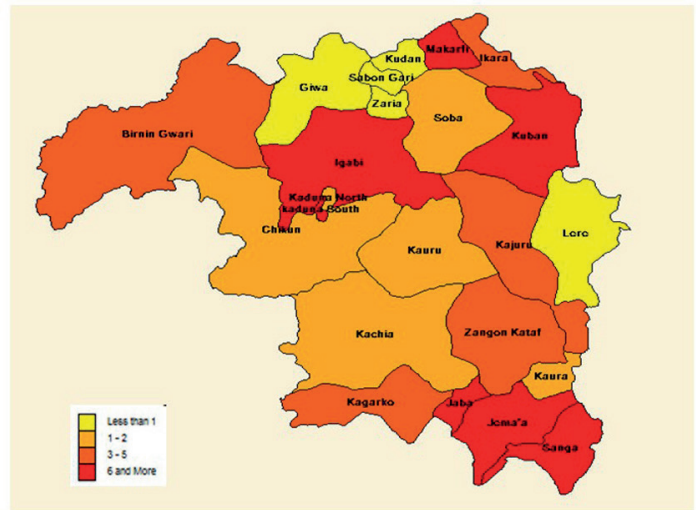

Figure 3: Map of Kaduna state showing percentage distribution of Malaria cases across its LGAs, 2014. 
ISDS 2016 Conference Abstracts

\section{Keywords}

Malaria; Surveillance; Evaluation; Kaduna

\section{Acknowledgments}

The authors gratefully acknowledged the support of the Kaduna state malaria control program through its program manager Mr. Ibrahim Sale and its monitoring and evaluation (M\&E) officer Mrs. Sakina Maikudi. All malaria focal persons of the state.

\section{References}

1. Organization WH. Background brief on the proposed targets and estimated costs of implementation of the draft global technical strategy for malaria (2016-2030): WHO; 2014 [3/20/2016].

2. Nnebue CC, Onwasigwe CN, Adogu POU, Onyeonoro UU. Awareness and knowledge of disease surveillance and notification by healthcare workers and availability of facility records in Anambra state, Nigeria. Nigerian Medical Journal : Journal of the Nigeria Medical Association. 2012;53(4):220-5.

3. German RR, Lee L, Horan J, Milstein R, Pertowski C, Waller M. Updated guidelines for evaluating public health surveillance systems. MMWR Recomm Rep. 2001;50(1-35).

4. Declich S, Carter AO. Public health surveillance: historical origins, methods and evaluation. Bulletin of the World Health Organization. 1994;72(2):285.

\section{*Baffa S. Ibrahim}

E-mail: baffasule@gmail.com 